\title{
Formation and Characterization of Sulfamethoxazole-Trimethoprim Cocrystal by Milling Process
}

\author{
Erizal Zaini $^{1 *}$, Yeyet C. Sumirtapura ${ }^{2}$, Auzal Halim ${ }^{1}$, Lili Fitriani ${ }^{1}$, Sundani N. Soewandhi ${ }^{2}$ \\ ${ }^{1}$ Department of Pharmaceutics, Faculty of Pharmacy, Andalas University, Padang, West Sumatera Indonesia. \\ ${ }^{2}$ Research Group of Pharmaceutics, School of Pharmacy, Bandung Institute of Technology, Bandung, West Java, Indonesia.
}

\begin{tabular}{l} 
ARTICLE INFO \\
\hline Article history: \\
Received on: 19/07/2017 \\
Accepted on: 08/11/2017 \\
Available online: 30/12/2017 \\
\hline Key words: \\
Co-crystal, trimethoprim, \\
sulfamethoxazole, milling \\
process.
\end{tabular}

\begin{abstract}
The purpose of this study was to describe and characterize co-crystal formation of sulfamethoxazole and trimethoprim. The co-crystal formation was carried out by solid state milling process. Co-crystal by solution cocrystallization and physical mixture were also prepared as a comparison. The potential co-crystalline phase was characterized by powder X-ray Diffraction (PXRD), thermal analysis differential scanning calorimetry (DSC), scanning electron microscope (SEM), and Fourier transform Infrared (FT-IR) spectroscopy. Effect of milling time on formation of co-crystal sulfamethoxazole and trimethoprim was investigated by DSC and PXRD analysis. The study showed that the milling process of sulfamethoxazole and trimethoprim in equimolar yielded the co-crystal. Intact co-crystal was obtained by solution co-crystallization with methanol. Prolongation of milling time accelerated the formation of co-crystal. Physical characterization showed that sulfamethoxazole and trimethoprim co-crystal demonstrated a unique powder X-ray diffraction, thermal and spectroscopic properties. The study concludes that the milling process induces equimolar co-crystal formation between sulfamethoxazole and trimethoprim. The transformation to co-crystalline phase is affected by milling time.
\end{abstract}

\section{INTRODUCTION}

Sulfamethoxazole and trimethoprim are synthetic and broad-spectrum antimicrobial agents that have been used clinically in combination to prevent microbial resistance. They are a synergic combination and act as an inhibitor of bacterial dihydrofolate reductase. Though newer antibacterial and antimicrobial drugs have supplanted this combination, these drugs are still used due to their low cost relatively and ability to act against common bacterial diseases efficiently (Hughes et al., 1974). Co-crystal is defined as a material which contains two or more discrete molecular entities in the crystal lattice (Kim et al., 2013). Our previous study has proven that sulfamethoxazole and trimethoprim could interact to form a co-crystal by hydrogen

* Corresponding Author

Erizal Zaini, Department of Pharmaceutics, Faculty of Pharmacy,

Andalas University, Padang, West Sumatera, Indonesia.

Email:erizal.ffua@gmail.com bonding in solid state by using solution co-crystallization with methanol. The 2-aminopyrimidine group of the trimethoprim is hydrogen bonded to the $\mathrm{N}$ (heteroatom)-C-NH(sulfonamide) moiety of sulfamethoxazole (Caira, 2007; Zaini et al., 2010). The solid state form of drug substances significantly affects physical properties such as solubility, dissolution rate, stability and bioavailability (Huang and Tong, 2004). Pharmaceutical co-crystal has gained more interest from pharmaceutical industries because they offer multiple opportunities for modification of the chemical and or physical properties of drug substances without making or breaking any covalent bonds. Indeed, many studies have reported the evident advantages of physicochemical properties of pharmaceutical co-crystal from pure drugs (Hiendrawan et al., 2016; Masuda et al., 2012; Maeno et al., 2014). Pharmaceutical co-crystal can be prepared by several methods. Recently, the preparation of co-crystal is mainly achieved by solution cocrystallization approach such as solvent evaporation and antisolvent addition (Zhang et al., 2007). 
In addition, crystallization from melting process and solid state grinding has also been employed (Berry et al., 2008; Chadwick et al., 2007). Shan et al. (2002) has modified this solid state grinding method by adding a small amount of appropriate solvent to wet the solids during grinding. This method is known with solvent-drop grinding and recognized as an alternative method to prepare the co-crystal.

The aims of the present study were to investigate the possibility of co-crystal formation between sulfamethoxazole and trimethoprim by milling process and to observe the effect of milling time on the formation of co-crystal. In addition, this study also carried out the formation of co-crystal by solution the cocrystallization method for comparative evaluation of co-crystal formation

\section{MATERIALS AND METHODS}

\section{Materials}

Sulfamethoxazole (SMZ) and trimethoprim (TMP) were kindly donated by PT. Pyridam (imported from Virchow Lab. India, batch no. 09150307 and Shouguang Fukang Pharm. Co. Ltd. batch no. 200703342, respectively). Potassium bromide crystal and methanol were produced by Merck, Germany. All materials were used as received

\section{Methods}

\section{Preparation of co-crystal of sulfamethoxazole-trimethoprim}

Co-crystal of sulfamethoxazole and trimethoprim were prepared in equimolar ratio by solid state milling method. Another co-crystal prepared by solution co-crystallization (SC) and the physical mixture (PM) of SMZ-TMP were used as comparison. Co-crystal produced by solid state milling was prepared by using Retsch Mühle apparatus (Germany) for 10, 20 and 30 minutes. Meanwhile, co-crystal by SC method was produced by dissolving in small amount of methanol at ambient temperature and then slowly evaporated at room temperature during 24 hours to promote co-crystallization. The physical mixture was prepared in a glass vial and underwent vortex mixing for 5 minutes. All samples were kept in a desiccator.

\section{Powder X-ray diffraction (PXRD)}

The PXRD patterns of intact materials, physical mixture and co-crystal were examined using Philips PW 1710 diffractometer (Philips, Holland). The experiments were conducted as following condition: $\mathrm{CuK} \alpha$ as radiation source, current at 30 $\mathrm{mA}$ and generator voltage $40 \mathrm{kV}$. The instrument was set over range $2 \theta$ of $5-40^{\circ}$ with a step size of 0.01 , and $0.5 \mathrm{sec} / \mathrm{step}$ with receiving slit $0.2 \mathrm{~mm}$.

\section{Differential Scanning Calorimetry (DSC)}

Approximately 1-4 mg of intact materials, physical mixture and co-crystal were placed in a pin-holed aluminum crimped pan to perform thermal analysis (Shimadzu Instrument Inc. DSC-60, Japan). Prior to measurement, the heating rate was maintained at $10^{\circ} \mathrm{C} / \mathrm{min}$ and temperature range was set at 30 $200^{\circ} \mathrm{C}$. Samples were purged with nitrogen gas at $60 \mathrm{~mL} / \mathrm{min}$ throughout the analysis.

\section{Scanning electron microscopy (SEM)}

The micrographs of crystal habit from intact materials, physical mixture and co-crystal were taken using SEM apparatus (JEOL model JSM-6360LA, Tokyo, Japan). The specimen was mounted on a metal stub with double sided adhesive tape and coated under vacuum with gold-palladium (Au 80\% and $\mathrm{Pd} 20 \%$ ) prior to observation.

\section{Fourier transform infrared (FT-IR) spectroscopy}

The infrared spectra of intact materials, physical mixture and co-crystal were recorded by FT-IR spectrophotometer (JASCO model FT-IR-4200 type A) using potassium bromide pellet over a range of $400-4000 \mathrm{~cm}^{-1}$.

\section{RESULTS}

\section{Powder X-ray diffraction (PXRD) analysis}

$X$-ray powder diffraction patterns of co-crystallization reaction products of intact sulfamethoxazole and trimethoprim can be seen in Figure 1A-B, while the diffractogram of its physical mixture is shown in Figure 2C. The diffraction pattern of cocrystal of sulfamethoxazole and trimethoprim shows some new and typical diffraction peaks at $2 \theta: 7.32 ; 11.5 ; 16.90 ; 19.15$ and $24^{\circ}$ (Figure 1D). On the other hand, diffraction pattern of the physical mixture after the milling process with variation time of 10,20 and 30 minutes at $2 \Theta$ are at $7.2 ; 9.58 ; 14.40 ; 16.77 ; 19.10$; and $23.00^{\circ}$ which demonstrates similar pattern with co-crystal phase from slow co-crystallization (Figure 2.).

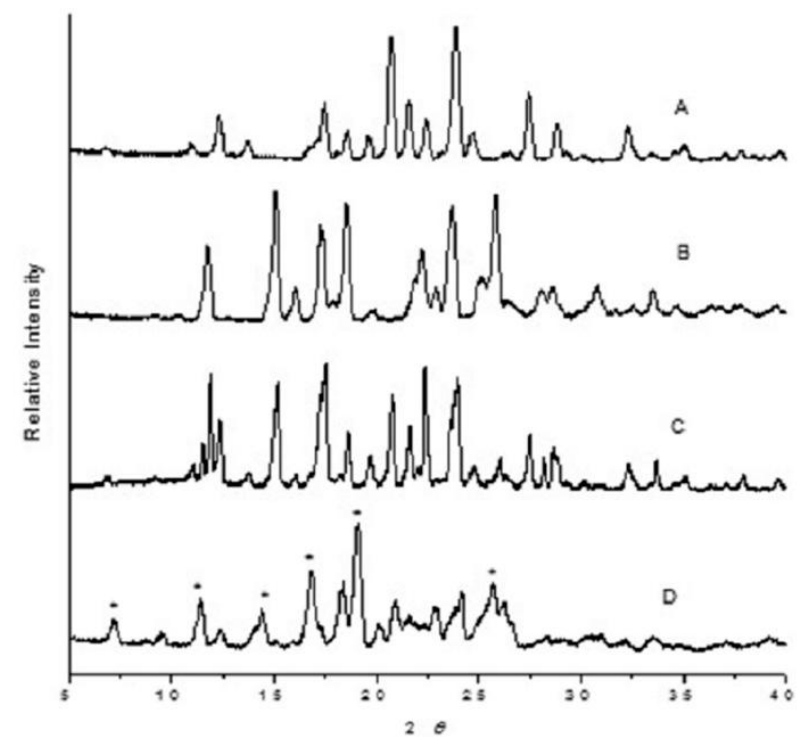

Fig. 1: Powder X-ray Diffractogram A) intact sulfamethoxazole, B) intact trimethoprim, C) physical mixture equimolar of sulfamethoxazole and trimethoprim and D) co-crystal of sulfamethoxazole and trimethoprim equimolar by cocrystalization technique from methanol. 


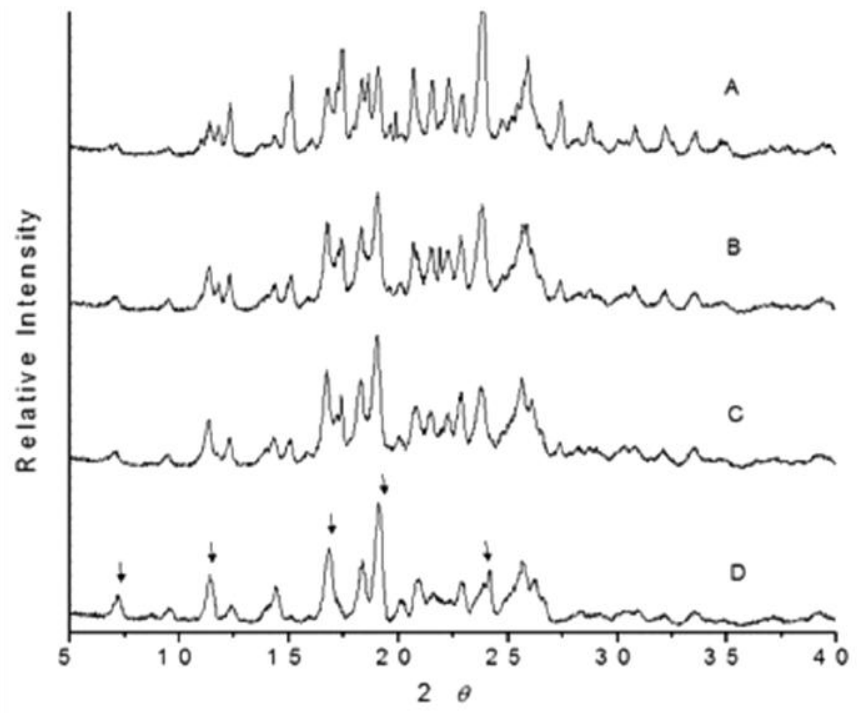

Fig. 2: Powder X-ray Diffractogram A) co-crystal by solid state milling for 10 min, B) $20 \mathrm{~min}$, C) $30 \mathrm{~min}$ and D) co-crystal of sulfamethoxazole and trimethoprim equimolar by cocrystalization technique from methanol.

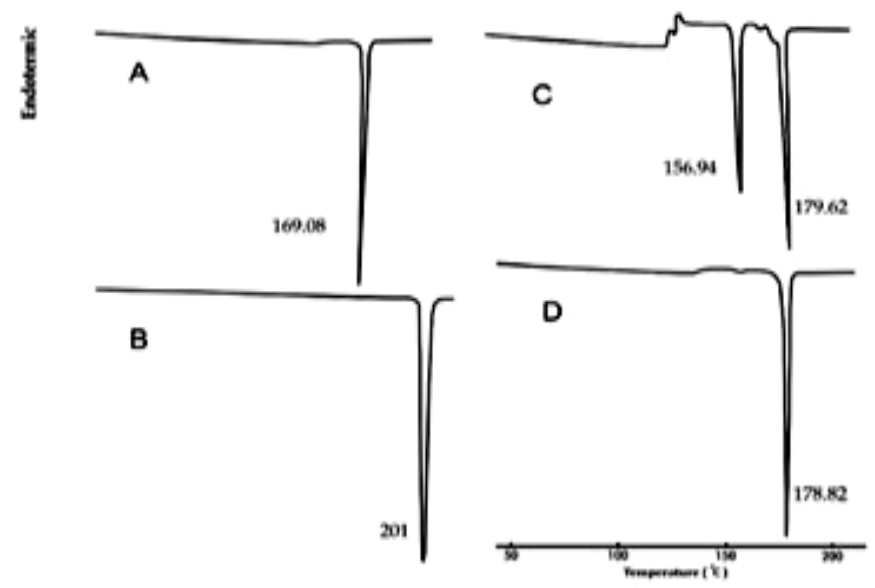

Fig. 3: DSC thermogram A) intact sulfamethoxazole, B) intact trimethoprim, C) physical mixture equimolar of sulfamethoxazole and trimethoprim and, D) co-crystal by co-crystallization technique from methanol.

\section{Differential scanning calorimetry (DSC) analysis}

Changes in thermal behavior of co-crystal of sulfamethoxazole and trimethoprim were shown in Figure 3 and 4. Intact sulfamethoxazole and trimethoprim crystals showed endothermic peaks due to the fusion at $169.8{ }^{\circ} \mathrm{C}$ and $201{ }^{\circ} \mathrm{C}$, respectively (Figure 3A-B). The physical mixture shows three endothermic peaks at $138,156.94$ and $179.62{ }^{\circ} \mathrm{C}$. The DSC thermogram of co-crystal from solution co-crystallization with methanol shows only one endothermic peak at $178.82{ }^{\circ} \mathrm{C}$ (Figure $3 \mathrm{D})$. The mixture of sulfamethoxazole and trimethoprim milled in different duration induced the formation of a new co-crystalline phase. This formation is indicated by endothermic peak at 178.53 in DSC thermogram (Figure 4).

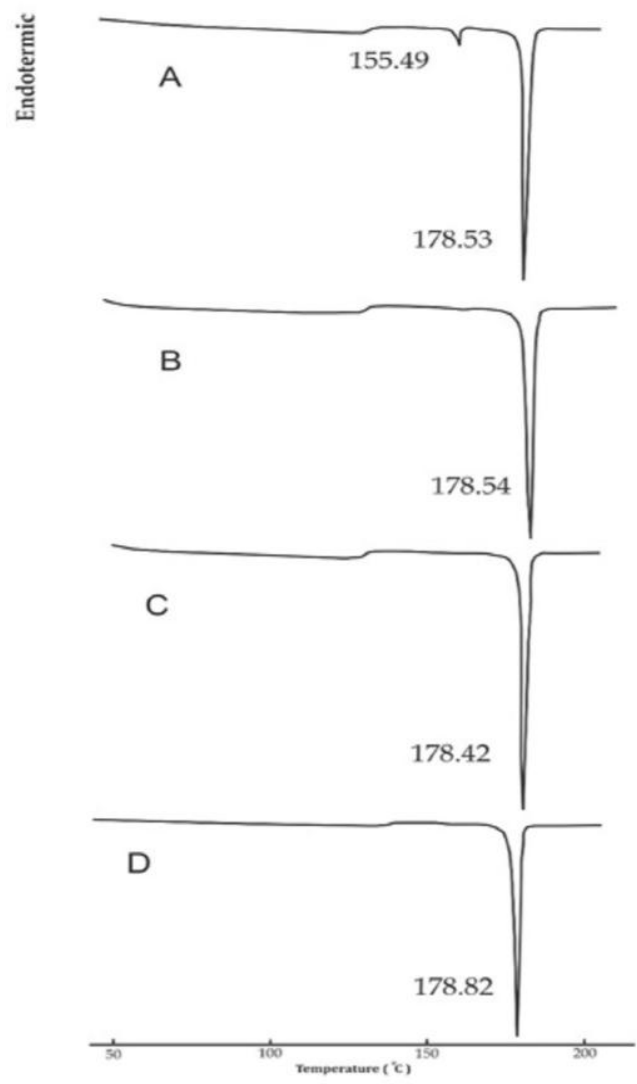

Fig. 4: DSC thermogram A) co-crystal by solid state milling for $10 \mathrm{~min}$, B) 20 min, C) $30 \mathrm{~min}$ and D) co-crystal of sulfamethoxazole and trimethoprim equimolar by cocrystalization technique from methanol.

\section{Scanning electron microscopy (SEM) analysis}

SEM micrographs (Figure 5. A-B) of intact sulfamethoxazole and trimethoprim shows different particle size of those materials. Figure 5C shows the habit of co-crystal prepared by milling process for 30 minutes, while Figure 5D shows the habit of co-crystal prepared by solution co-crystalization obtained within 24 hours that appears as needle-shaped crystals.
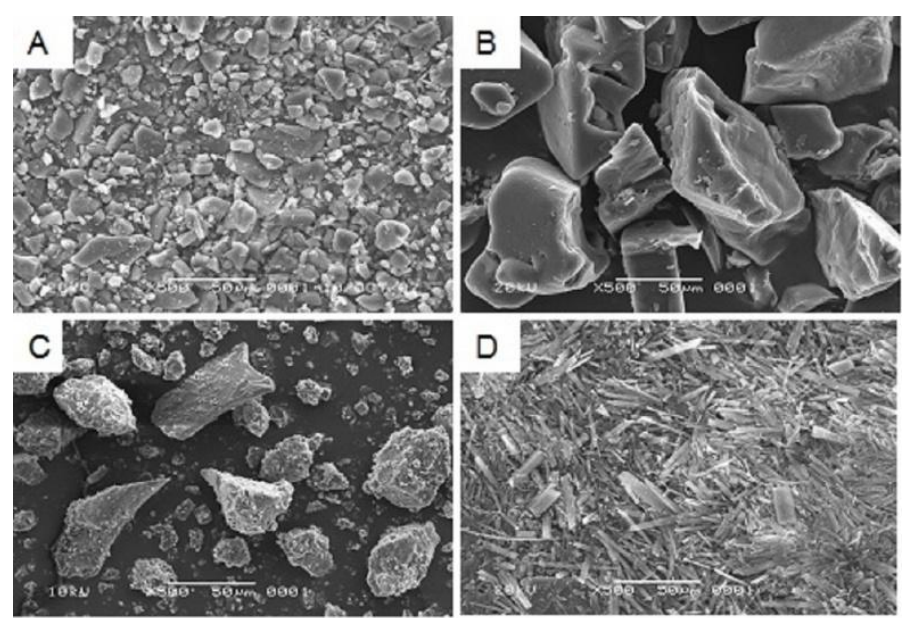

Fig. 5: Scanning Electron Micrographs A) intact sulfamethoxazole, B) intact trimethoprim, C) co-crystal sulfamethoxazole and trimethoprim by solid state milling and D) co-crystal by cocrystalization technique from methanol. 


\section{Fourier transform infrared (FT-IR) spectroscopy analysis}

Figure 6 shows molecular interaction between sulfamethoxazole and trimethoprim in milled mixture was investigated by FT-IR spectroscopy. The spectral differences between the co-crystal and intact sulfamethoxazole and intact trimethoprim are seen at wave number around 3000 to $4000 \mathrm{~cm}^{-1}$. This spectrum is particularly noticed at wave numbers $3400 \mathrm{~cm}^{-1}$, in which the N-H strain is shifted to a lower wave number.
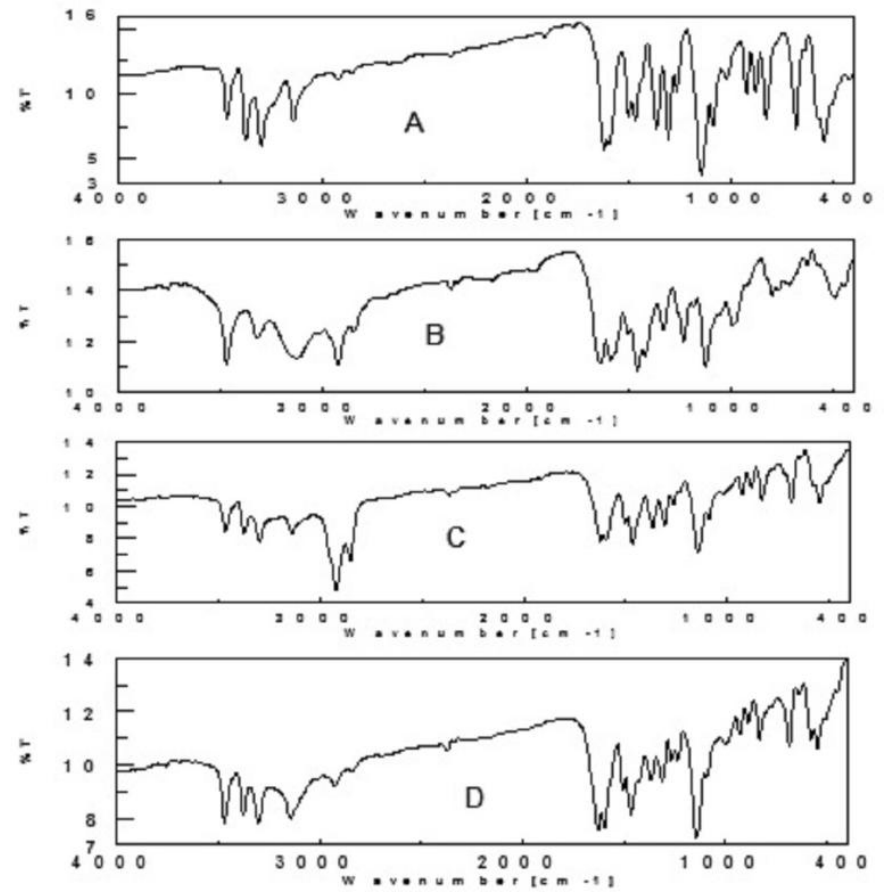

Fig. 6: FT-IR Spectra A) intact sulfamethoxazole, B) intact trimethoprim, C) physical mixture equimolar of sulfamethoxazole and trimethoprim and, D) cocrystal by solid state grinding during $30 \mathrm{~min}$.

\section{DISCUSSION}

Milling is one of essential processes in manufacturing pharmaceutical preparations, especially to reduce particle size of active ingredients and excipients (Loh et al., 2015). Co-milling of solids have been reported to cause solid phase reactions, either physically or chemically (Boldyrev, 2004; Hu et al., 2014). Cocrystal phase formation between two crystalline solids by solid state milling method has been recognized as an alternative method (Friscic and Jones, 2009; Lin, 2016). In addition, milling can be conducted by mixing two components to form co-crystal, milling either manually with a mortar and pestle, or mechanically using a ball mill and vibratory mill. The milling process has been utilized to induce the co-crystal phase formation of carbamazepine with nicotinamide and saccharin (Chieng et al., 2009; Jayasankar et al., 2006).

PXRD is a foremost method to characterize the formation of a new crystalline phase in solid state. The diffraction pattern of co-crystal should be distinct from the superimposition of each compounds if a true co-crystal has been formed between two solid phases. Our previous study showed that the co-crystal of sulfamethoxazole and trimethoprim had been successfully formed through solution co-crystallization (Zaini et al., 2010). This cocrystal was used in the present study as a reference. The PXRD pattern of each intact drugs demonstrates a crystalline solid marked by typical interference peaks at various diffraction angles (20) shown in Fig. 1A-B. However, PXRD pattern of physical mixture (Fig 1C) shows all characteristic interference peaks of sulfamethoxazole and trimethoprim, which indicates a superimposition of the two components. The duration of milling time influenced the intensity of some interference peaks, particularly at $2 \theta: 16.77$ and $19.10^{\circ}$. At these points, the interference peaks increase and broaden, which indicates the amorphization of partial solid phase interaction (Fig. 2A-C). The diffraction pattern of co-crystal shows some new and typical interference peaks at $2 \theta: 7.32 ; 11.5 ; 16.90 ; 19.15$ and $24^{\circ}$. This indicated the physical interaction between sulfamethoxazole and trimethoprim and the formation of a new crystalline phase, known as co-crystalline phase (molecular compounds or molecular complexes) in material sciences (Masuda et al., 2012; Zhang at al, 2007).

Thermal analysis is one of the most widely used methods for studying the solid state interaction of pharmaceutical substances. In particular, Differential Scanning Calorimetry (DSC) study is an important analytical tool in characterization of solidstate interaction between two or more drug substances. Among available methods, DSC is used for investigating the thermodynamic changes which occur while heating a substance. These include recrystallization, melting and de-solvation which can all show endothermic or exothermic peaks on the DSC curves (Giron, 2002). The present study showed that the milled sample in solid state for different duration caused the formation of co-crystal (Fig. 4A-C). The DSC thermogram of physical mixture shows three endothermic peaks, at $138{ }^{\circ} \mathrm{C}$ and $156.94{ }^{\circ} \mathrm{C}$ which are attributed to fusion of eutectic mixture, and at $179.62^{\circ} \mathrm{C}$ due to the fusion of co-crystal. Meanwhile, the exothermic peaks at $140^{\circ} \mathrm{C}$ is attributed to recrystallization of mixture from melting. On the other hand, the co-crystal by milling method induced the formation of a new co-crystalline phase, indicated by endothermic peak at $178.53{ }^{\circ} \mathrm{C}$ in DSC thermogram. The DSC peak area of eutectic fusion decreases by prolongation of grinding time, indicating the milling-induced progress of sulfamethoxazole-trimethoprim cocrystal formation (Chieng et al., 2009). In similar, the DSC thermogram of co-crystal from solution co-crystallization of methanol also shows only one endothermic peak at $178.82{ }^{\circ} \mathrm{C}$, which indicates the formation of new crystalline phase of cocrystal between sulfamethoxazole and trimethoprim (Zaini at al, 2010).

Scanning Electron Microscopy (SEM) analysis depicts a decrease of particle size and drugs is embedded each other to form coherence particles. As a reference to compare the possibility of co-crystal formation between sulfamethoxazole and trimethoprim by solid state grinding, co-crystal was also prepared by solution co-crystallization technique by using methanol. The reaction 
between the solid phases can be mediated by an intermediate liquid phase, or requires the destruction of the crystalline structure prior to the formation of a new crystalline phase. This condition is often observed in solid state reactions which is activated by mechanical energy. The molecular reactions of sulfamethoxazole and trimethoprim crystals may occur on the surface of the crystal or require molecular diffusion within or between crystal lattices. The mechanical energy generated by the grinding process of two or more solids will cause molecular diffusion along the crystal fracture and an increase on the surface area of the crystal, thereby allowing solid-solid interaction to occur (Braga et al., 2005).

FT-IR spectroscopy analysis is used to characterize the complex of two compounds in solid state which can be linked by hydrogen bonding (Bugay, 2001). As seen in Figure 6, there is a shift of N-H strain which indicates the formation of hydrogen bond as a consequence of co-crystal phase formation between sulfamethoxazole and trimethoprim (Caira, 2007). The interaction between sulfamethoxazole and trimethoprim may occur as a result of $\mathrm{pKa}$ difference, in which a molecule of trimethoprim is bonded with one molecule of sulfamethoxazole. This bonding may present through hydrogen bond of 2-aminopirimidine group in trimethoprim with group $\mathrm{N}$ (heteroatom) - $\mathrm{C}-\mathrm{NH}$ (sulfonamide) of sulfamethoxazole (Bettinetti, G., and Giordano, F., 1988).

\section{CONCLUSION}

This study confirms that solid state milling method induce the formation of co-crystal from sulfamethoxazole and trimethoprim. The PXRD pattern of the co-crystal demonstrates a new crystalline phase. The co-crystal is formed due to mechanical stress-induced interaction in a solid state between the two substances.

\section{ACKNOWLEDGEMENTS}

We would like to thank Indonesia National Agency of Drug and Food Control for providing thermal DSC analysis.

\section{Financial support and sponsorship: Nil.}

Conflict of Interests: There are no conflicts of interest.

\section{REFERENCES}

Berry DJ, Seaton CC, Clegg W, Harrington RW, Coles SJ, Horton PN, Hursthouse MB, Storey R, Jones W, Friscic T, Blagden N. Applying hot-stage microscopy to co-crystal screening: a study of nicotinamide with seven active pharmaceutical ingredients. Cryst Growth Des. 2008; 8(5):1697-712.

Bettinetti G, Giordano F. Interaction between trimethoprim and some sulfa drugs. Drug Dev Ind Pharm. 1988;14(4):431-49.

Boldyrev VV. Mechanochemical modification and synthesis of drugs. J Mater Sci. 2004; 39(16-17):5117-20.

Braga D, D'Addario D, Giaffreda SL, Maini L, Polito M, Grepioni F. Intra-solid and inter-solid reactions of molecular crystals: a green route to crystal engineering. Top Curr Chem. 2005(254): 71- 94

Bugay DE. Characterization of the solid-state: spectroscopic techniques. Adv Drug Del Rev. 2001; 48(1):43-65.
Caira MR. Sulfa drugs as model cocrystal formers. Mol Pharm. 2007;4(3):310-6.

Chadwick K, Davey R, Cross W. How does grinding produce co-crystals? Insights from the case of benzophenone and diphenylamine. CrystEngComm. 2007;9(9):732-4.

Chieng N, Hubert M, Saville D, Rades T, Aaltonen J. Formation kinetics and stability of carbamazepine- nicotinamide cocrystals prepared by mechanical activation. Cryst Growth Des. 2009; 9(5):2377-86.

Friscic T, Jones W. Recent advances in understanding the mechanism of cocrystal formation via grinding. Cryst Growth Des. 2009;9(3):1621-37.

Giron D. Applications of thermal analysis and coupled techniques in pharmaceutical industry. J Therm Anal Calorim. 2002; 68(2):335-57.

Hiendrawan S, Veriansyah B, Widjojokusumo E, Soewandhi SN, Wikarsa S, Tjandrawinata RR. Physicochemical and mechanical properties of paracetamol cocrystal with 5-nitroisophthalic acid. Int $\mathbf{J}$ Pharm. 2016;497(1):106-13.

Hu Y, Gniado K, Erxleben A, McArdle P. Mechanochemical reaction of sulfathiazole with carboxylic acids: Formation of a cocrystal, a salt, and coamorphous solids. Cryst Growth Des. 2014;14(2):803-13.

Huang LF, Tong WQ. Impact of solid state properties on developability assessment of drug candidates. Adv Drug Del Rev. 2004;56(3):321-34.

Hughes WT, McNabb PC, Makres TD, Feldman S. Efficacy of trimethoprim and sulfamethoxazole in the prevention and treatment of Pneumocystis carinii pneumonitis. Antimicrob Agents Chemother. 1974;5(3):289-93.

Jayasankar A, Somwangthanaroj A, Shao ZJ, RodríguezHornedo N. Cocrystal formation during cogrinding and storage is mediated by amorphous phase. Pharm Res. 2006; 23(10):2381-92.

Kim S, Li Z, Tseng YC, Nar H, Spinelli E, Varsolona R, Reeves JT, Lee H, Song JJ, Smoliga J, Yee N. Development and characterization of a cocrystal as a viable solid form for an active pharmaceutical ingredient. Org Process Res Dev. 2013;17(3):540-8.

Lin SY. Mechanochemical approaches to pharmaceutical cocrystal formation and stability analysis. Curr Pharm Des. 2016;22(32):5001-18.

Loh ZH, Samanta AK, Heng PW. Overview of milling techniques for improving the solubility of poorly water-soluble drugs. Asian J Pharm Sci. 2015;10(4):255-74.

Maeno Y, Fukami T, Kawahata M, Yamaguchi K, Tagami T, Ozeki T, Suzuki T, Tomono K. Novel pharmaceutical cocrystal consisting of paracetamol and trimethylglycine, a new promising cocrystal former. Int J Pharm. 2014; 473(1): 179-86.

Masuda T, Yoshihashi Y, Yonemochi E, Fujii K, Uekusa H, Terada K. Cocrystallization and amorphization induced by drug-excipient interaction improves the physical properties of acyclovir. Int $\mathrm{J}$ Pharm. 2012; 422(1): 160-9.

Shan N, Toda F, Jones W. Mechanochemistry and co-crystal formation: effect of solvent on reaction kinetics. Chem Comm. 2002; (20): 2372-3.

Zaini E, Sumirtapura YC, Soewandhi SN, Halim A, Uekusa H, Fujii K. Cocrystalline phase transformation of binary mixture of trimethoprim and sulfamethoxazole by slurry technique. Asian J Pharm Clin Res. 2010; 3(4):26-9.

Zhang GG, Henry RF, Borchardt TB, Lou X. Efficient co crystal screening using solution - mediated phase transformation. J Pharm Sci. 2007; 96(5):990-5.

\section{How to cite this article:}

Zaini E, Sumirtapura YC, Halim A, Fitriani L, Soewandhi SN. Formation and Characterization of Sulfamethoxazole-Trimethoprim Cocrystal by Milling Process. J App Pharm Sci, 2017; 7 (12): 169173. 